\title{
Desenvolvimento de Objeto de Aprendizagem, baseado em Especificações de Normatização SCORM, para o Caso de Suporte à Aprendizagem de Funções.
}

\author{
Carlos Eduardo Milanezi Vieira \\ Evanio Ramos Nicoleit
}

Departamento de Ciência da Computação - Universidade do Extremo Sul Catarinense

$$
\text { cadumil@gmail.com, ern@unesc.net }
$$

\section{Resumo:}

Este trabalho descreve o desenvolvimento de um Objeto de Aprendizagem direcionado à área da matemática usando recursos de multimídia como figuras, animações e sistemas interativos para a compreensão do assunto. $\mathrm{O}$ trabalho baseia-se no Padrão SCORM para Materiais Instrucionais e Objetos de Aprendizagem. Também explora a criação das páginas Web que compõem o objeto e o desenvolvimento da interatividade deste com o aluno - obtida a partir da linguagem Java com recursos de criação de gráficos e interpretação automática das funções matemáticas. Aspectos pedagógicos acerca da produção e utilização dos objetos de aprendizagem também são discutidos.

Palavras-Chave: Objeto de aprendizagem, Padrão SCORM, Educação a Distância.

Title: Development of learning object, based on specifications of SCORM standard, for the case of supporting to functions learning.

\begin{abstract}
This paper describes the development of a Learning Object directed to the mathematics using resources such as figures, animation and interactive systems for the understanding of the matter. The work is based on SCORM Standard for Educational Materials and Learning Objects. Also it explores the creation of Web pages that compose the Learning Object and the development of the interactivity of this with the student - obtained from the Java language resources for the creation of graphs and automatic interpretation of the mathematical functions. Pedagogical aspects concerning the production and use of Learning Objects are also discussed.
\end{abstract}

Keywords: Learning Objects, SCORM standard, e-learning.

\section{Introdução}

A educação superior historicamente mostra que as ciências exatas são uma área do conhecimento onde os alunos apresentam dificuldades no processo de aprendizagem. Constantemente ocorrem desníveis na formação destes alunos, além das diferentes exigências dos programas curriculares agravarem o problema (ZANETTE, NICOLEIT e GIACOMAZZO, 2006).

As Tecnologias da Informação e Comunicação (TICs) vêm sendo cada vez mais utilizadas na educação. O uso das TICs no ensino de matemática possibilita novas práticas pedagógicas. Permite, pelo uso de seus recursos tecnológicos, pesquisar, fazer antecipações e simulações, confirmar idéias prévias, experimentar, criar soluções e construir novas formas de representação mental (ZANETTE, NICOLEIT e GIACOMAZZO, 2006). 
Objetos de aprendizagem (OA) vêm se mostrando como uma alternativa aos professores no apoio ao processo ensino-aprendizagem. O presente trabalho discute $\mathrm{O}$ desenvolvimento de um OA para ensino de funções, visando a superação das dificuldades na apropriação dos conhecimentos científicos matemáticos.

\title{
2. Objetos de Aprendizagem (OA)
}

Conforme Tarouco, Fabre e Tamusiunas (2003), os objetos de aprendizagem são quaisquer recursos que possam ajudar no processo de ensino e ser reutilizados em diversos contextos. Uma definição que aproxima os objetos de aprendizagem da informática argumenta que estes objetos são recursos digitais que podem ser utilizados para dar suporte ao ensino, e que são construídos de forma a dividir o conteúdo em pequenos módulos reutilizáveis em diversos ambientes seguindo os princípios da orientação a objetos (WILEY, 2001).

\begin{abstract}
Um objeto de aprendizagem tem como função atuar como recurso didático interativo, abrangendo um determinado segmento de uma disciplina e agrupando diversos tipos de dados como imagens, textos, áudios, vídeos, exercícios, e tudo o que pode auxiliar o processo de aprendizagem. Pode ser utilizado - tanto no ambiente de aula, quanto na Educação à Distância (MACHADO e SILVA, 2005).
\end{abstract}

Isto significa tratar os objetos como elementos preparados a fim de dar apoio ao ensino e delimitando de maneira clara os tipos de recursos que eles podem utilizar.

Pode se portanto definir com base nestes conceitos um objeto de aprendizagem. Objeto de Aprendizagem é um recurso digital que pode ser utilizado como auxílio ao processo de ensino-aprendizagem, e que tem a capacidade de ser reutilizado em vários contextos de maneira a facilitar a apropriação do conhecimento.

Os objetos devem ser concebidos com a premissa de serem facilitadores na construção do conhecimento, e devem servir como instrumento para que o aluno construa o seu entendimento sobre o assunto que está sendo abordado.

Pode-se utilizar um objeto de aprendizagem, por exemplo, para realizar simulações de experiências e atividades práticas. Ele permite que o aluno teste, de maneira prática e interativa, inúmeras possibilidades do exercício proposto, que, se tivesse sido estudado apenas teoricamente, não estimularia tanto a aprendizagem do conteúdo (MACHADO e SILVA, 2005).

Desta forma, os OAs devem ser projetados de forma a integrar-se com outros semelhantes e que esta junção possibilite a criação de contextos maiores. Ou seja, eles são módulos que tem o objetivo de se ligarem e possibilitar a construção do conhecimento (HODGINS e CONNER, 2001). É necessário que hajam características de padronização que permitam esta integração. As características são as seguintes: reusabilidade; autonomia; interatividade; interoperabilidade e; facilidade de busca.

Para que as características descritas anteriormente sejam contempladas, o desenvolvimento precisa seguir uma padronização de parâmetros no desenvolvimento de OAs. A adoção de padrões possibilita a uniformização na produção de elementos (OAs) com características unificadas e simplificadas segundo um modelo preestabelecido, desde que construídos seguindo as normatizações definidas pelo padrão adotado (PASSARINI, 2003). Os objetos de aprendizagem devem ser desenvolvidos de maneira a prover compatibilidade com outros objetos. Atualmente existem diversas especificações que normatizam o desenvolvimento dos objetos. Entre as principais especificações podemos destacar o Instructional Managment System (IMS) Learning Design, o Sharable Content Objetct Reference Model (SCORM) e o Aviation Industry 


\section{CBT (Computer-Based Training) Committe (AICC).}

O SCORM é desenvolvido de modo a incorporar diversas especificações de objetos de ensino e, desta forma, permitir sua compatibilidade com os mais diversos LMS existentes (BAILEY, 2005). O padrão SCORM é uma junção de características das especificações de objetos de aprendizagem realizadas por outras entidades, resultando em um padrão mais geral e compatível.

O conjunto de especificações do SCORM descreve como o conteúdo do OA é criado e encapsulado, como ele é apresentado para um aluno e como avalia a sua evolução (BAILEY, 2005). Estas descrições estão colocadas em três diferentes publicações que compõe o padrão SCORM na sua última versão: SCORM 2004. As três publicações são (DUTRA e TAROUCO, 2006):

a) Modelo de Agregação de Conteúdo: que especifica o dicionário de metadados, seu código Extensible Markup Language (XML) e também define como fazer a estruturação e o empacotamento do conteúdo do objeto;

b) Ambiente de Execução: responsável pela comunicação do objeto de aprendizagem com o LMS, ou seja, comunicações sobre o andamento do curso e sobre a evolução do aluno;

c) Seqüenciamento e Navegação: que orienta sobre como os objetos de aprendizagem devem ser seqüenciados por um LMS e quais as avaliações que habilitam o aluno a progredir no módulo (contexto, ou curso).

Há que se observar que o padrão SCORM é direcionado para o conteúdo do OA e na maneira como ele deve ser criado de modo a ser automatizado. Porém, não se aprofunda em outras questões do ensino, ficando restrito à interação direta do aluno com o OA (DUTRA e TAROUCO, 2006).

\section{Aspectos Pedagógicos}

A utilização de OAs possibilita ao aluno conhecer novas ferramentas, testar diferentes situações, arriscar, antecipar, compreender a relação entre causa e efeito de conceitos e fenômenos, para despertar a curiosidade e para resolver problemas (RIVED, 2006).

O uso das tecnologias da informação como instrumento para construção do conhecimento está em um processo de forte expansão, justamente por possibilitar às escolas a realização de experiências além da sala de aula. Entretanto, a utilização da tecnologia no ensino não deve ser feita de maneira ingênua e prematura, mas sim acompanhada de um estudo abrangente sobre como um sujeito adquire e constrói o conhecimento (FERREIRA et al, 2004).

Para que um objeto de aprendizagem tenha eficácia didática o educador deve procurar responder algumas questões:

\footnotetext{
"Deve-se definir, antecipadamente à criação dos objetos de aprendizagem, qual será o seu objetivo: O que ele vai ou não abordar? Com que profundidade? Qual enfoque adequado? Para que público? Qual a importância deste tópico para o conhecimento (disciplina e/ou curso) que se deseja transmitir? e Quais formas de Interatividade com o educando? Ainda deve-se planejar quais as metodologias e ferramentas aplicadas na construção do objeto de aprendizagem para que ele atinja os seus objetivos.” (BORGES; NAVARRO, 2005).
}

Ainda, conforme Borges e Navarro (2005), a elaboração de um OA parte da noção de quais resultados se deseja obter ao final da apreciação pelo aluno. Um objeto de aprendizagem, por si só, não irá responder a todas as exigências que a construção do 
conhecimento envolve. Porém, ele pode ser planejado de modo a assegurar o desenvolvimento gradativo das competências e habilidades do indivíduo. Este tipo de abordagem vislumbra o objeto de aprendizagem trabalhando de forma colaborativa com outros recursos educacionais.

O processo de aprendizagem agrega três fatores importantes: que o conteúdo seja estruturado de maneira compreensível; que o aluno tenha um conhecimento prévio que lhe permita entender o que será abordado; confiança do aluno em aceitar este tipo de ensino relacionando-o com o que ele já conhece previamente. Portanto, um objeto de aprendizagem pode ter sucesso no intuito de auxiliar na construção do conhecimento se for capaz de servir de ponte entre o conhecimento básico, que o aluno já possui, e o conhecimento avançado que se pretende agregar. Este objetivo pode ser alcançado com o uso de recursos visuais como animações e simulações que despertem a atenção do aluno e façam com que ele possa vivenciar o tema abordado (BORGES e NAVARRO, 2006).

Uma vantagem da utilização dos objetos de aprendizagem é que ele respeita o ritmo de aprendizagem de cada indivíduo, o que geralmente não ocorre na educação presencial (ZANETTE, NICOLEIT e GIACOMAZZO, 2006).

Observa-se que a utilização dos objetos de aprendizagem pode prover ao aluno um apoio na construção de conhecimentos e conceitos de maneira mais interativa e eficiente. Não há um modelo pedagógico único, uma fórmula pronta de se apresentar um conteúdo por meio de um OA. Desta maneira, a abordagem do tema fica a cargo da criatividade do educador. Todavia, é necessário que seja um conteúdo interessante. Constata-se que a construção e a utilização de um objeto de aprendizagem depende do planejamento do educador de modo a possibilitar a construção do conhecimento com o apoio de um conteúdo interativo e atraente.

\section{Recursos Tecnológicos}

O Java é uma importante ferramenta no desenvolvimento de objetos de aprendizagem, pois permite a inserção de gráficos interativos além de outros tipos de animações para internet, inclusive com recursos 3D (MUTINI, 2006). Segundo, Sahara e Brandão (2000) a integração do Java com a interface Web pode ser realizada utilizando um applet (ISOTANI, 2000).

O uso de gráficos para demonstrar o comportamento de certas situações é comum na matemática e auxilia no desenvolvimento de OAs. A biblioteca para a linguagem Java JFreeChart é um dos recursos que permite a criação de gráficos (GOMES, 2006). Os gráficos que serão disponibilizados no objeto de aprendizagem em questão são do tipo gráfico de linhas, que é baseado no plano cartesiano x e y.

Para a criação de animações, uma das ferramentas mais populares é o Macromedia Flash. Ele permite que o usuário crie suas figuras e anime-as (TAROUCO et al, 2004). Para este projeto foi utilizado o Macromedia Flash MX na versão de testes.

A comunicação do objeto de aprendizagem com um LMS é realizada pelo uso de uma Application Programming Interface - API, como designa o padrão SCORM, e segue as orientações do AICC e do LTSC. A API é um pequeno sistema escrito na linguagem JavaScript e que implementa algumas funções básicas para informar ao LMS se o objeto de aprendizagem foi iniciado com sucesso e quando ele é encerrado, por exemplo.

Neste projeto foi utilizado ainda o programa Dreamweaver MX na sua versão de teste para a montagem das páginas Web. 
Quanto ao layout das páginas, embora não haja dentro do SCORM nenhum padrão neste sentido, este foi desenvolvido seguindo as orientações de um padrão definido pelo RIVED.

\section{Desenvolvimento do Objeto de Aprendizagem}

A criação de um objeto de aprendizagem é uma tarefa que exige um trabalho colaborativo intenso. Tão importante quanto o conhecimento sobre as ferramentas de desenvolvimento computacional, é ter noção de como ocorre a construção do conhecimento; é pensar como professor, como aluno e como programador. A parte lógica do desenvolvimento é um tanto mais simples, neste caso, por se tratar de área conhecida para qual o desenvolvedor é preparado ao longo da sua rotina diária. Porém, organizar este conhecimento de forma a produzir algo atraente para aprendizes torna-se uma tarefa consideravelmente complexa. Constata-se portanto a necessidade de uma prática colaborativa e cooperativa com o auxílio de especialistas nas áreas de programação, de design, pedagógica, além de especialistas da área do conhecimento específico - neste caso, a matemática - do OA.

Para o desenvolvimento do objeto de aprendizagem, são consideradas permanentemente informações acerca de novas práticas pedagógicas, bem como tecnologias emergentes que possam dar suporte a evoluções sobre os OAs.

Internamente, o modelo de agregação de conteúdo do padrão SCORM é dividido em três partes - modelo de conteúdo; encapsulamento do conteúdo e; metadados. Cada qual com sua função definida, permitem criar um OA com informações completas sobre conteúdo, localização e distribuição.

O modelo de conteúdo define os recursos de baixo nível que podem ser utilizados na montagem de um elemento instrucional e como estes recursos podem ser agrupados em componentes maiores. O asset é o recurso digital mais básico que pode ser utilizado dentro do conteúdo instrucional do objeto de aprendizagem. Ele é formado por qualquer texto, animação, som, sistema interativo, imagem ou outros elementos que tenham relação com o que se deseja criar. O Shareable Content Object (SCO) representa uma associação de vários assets. Eles têm a capacidade de se comunicar com os LMS, podendo desta forma se associar com outros semelhantes para formar atividades mais complexas. Devem ser construídos de forma a ser unidades pequenas que possam ser reutilizadas em contextos diversos. A organização do conteúdo é dividida em dois grupos: atividades e recursos. Os recursos são formados por assets e SCOs. As atividades são como módulos ou tópicos do objeto de aprendizagem. Cada OA pode ser composto por várias atividades, e uma atividade por sua vez pode conter outras atividades ou simplesmente conter os recursos (ADL, 2006).

O encapsulamento de conteúdo garante a disponibilização do OA nos LMS para busca e acesso. O pacote é composto de dois elementos: o primeiro elemento é um arquivo XML, conhecido como manifesto, que descreve a estrutura do objeto e o seu conteúdo; o segundo elemento contém os arquivos multimídia propriamente ditos (animações, textos, html, applets Java, etc.). Os LMS utilizam as informações do manifesto para conhecer a organização do conteúdo do objeto.

$\mathrm{O}$ arquivo de metadados é o recurso utilizado para rotular o objeto instrucional desenvolvido. O esquema de criação dos metadados SCORM segue o que estabelece o IEEE - Learning Object Metadata, para garantir interoperabilidade com a maioria dos LMS. Os metadados têm por objetivo criar uma espécie de cabeçalho para o OA com informações padronizadas que facilitam a busca e acesso pelos sistemas LMS. 
Estas informações são referentes ao titulo do objeto, descrição do seu conteúdo, autoria, tempo de vida e uma série de outros dados (ADL, 2006).

O padrão SCORM orienta que o ambiente de execução dos objetos de aprendizagem sejam voltados para os LMS. No entanto, para que um conteúdo instrucional seja acessado e a sua evolução seja monitorada é necessário que o próprio objeto se comunique com o LMS e desta forma lhe forneça os subsídios necessários para o gerenciamento do conteúdo. Esta comunicação é feita pelo uso da API.

Neste contexto podemos considerar que a API implementada pelo OA localiza a outra implementada pelo LMS, e com isto se inicia a comunicação com a função Initialize(). Pode haver ainda outras funções, criadas para a troca de informações entre o objeto e o LMS, em casos onde este controle a evolução do aluno baseado nas avaliações programadas no OA. No entanto esta limitação segue as orientações dos designers pedagógicos. No caso deste objeto desenvolvido, não há limitações na progressão do aluno pelo conteúdo. Portanto a API do objeto apenas trabalha com a função Initialize() e Terminate(), esta última utilizada para encerrar a comunicação com o LMS.

O design pedagógico de objetos de aprendizagem requer a orientação de especialistas no tema abordado. O objeto de aprendizagem a que este trabalho se destina tem como objetivo dar suporte à disciplina de Cálculo Diferencial e Integral I. Foram convidados para definir o conteúdo pedagógico os professores da área da matemática e do Grupo de Pesquisa em Educação a Distância na Graduação da UNESC.

Em seguida são apresentadas e discutidas algumas telas que exemplificam o conceito pedagógico adotado no desenvolvimento. O RIVED define por padrão que o objeto de aprendizagem deve ter uma tela inicial com a qual se possa identificar a idéia do objeto. A Figura 1 mostra a capa do OA.

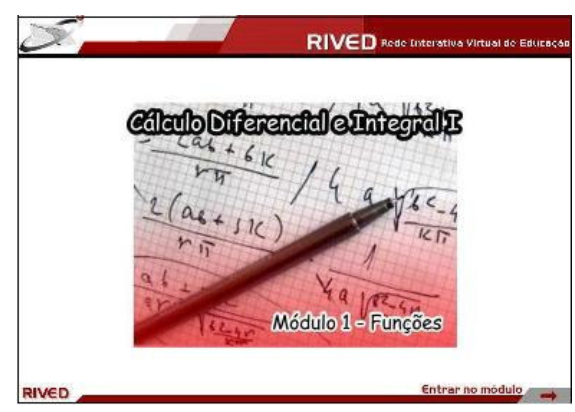

Figura 1 - Tela inicial do objeto de aprendizagem

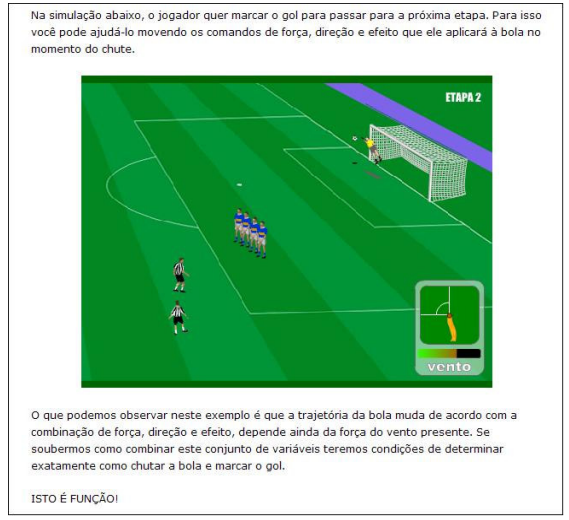

Figura 2 - Animação que exemplifica uma função.

Após a apresentação do objeto de aprendizagem o conteúdo do módulo é iniciado. O tema inicial são as funções. Para exemplificar o seu conceito, foi utilizada uma animação simulando uma situação cotidiana que é facilmente compreensível pelos aprendizes, como apresentado na Figura 2.

Além dos recursos de animação, também foram utilizadas figuras estáticas de personagens para apresentação de conceitos. A Figura 3 mostra uma destas imagens utilizadas no objeto.

Em determinados momentos da apresentação do conteúdo foram inseridos pequenos sistemas escritos em Java para possibilitar a interação do aluno com o OA. 
Nestas simulações o aluno pode modificar o valor de constantes de uma dada função apresentada e observar o seu comportamento conforme ocorre a variação de x. A Figura 4 exemplifica um applet Java. A ferramenta utilizada para a composição dos gráficos permite a representação de várias funções simultaneamente no plano cartesiano, como mostra a Figura 5.

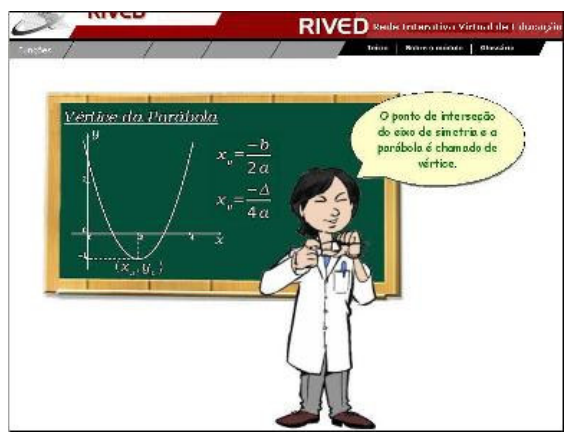

Figura 3 - Imagem de gráfico para exemplo do conceito abordado

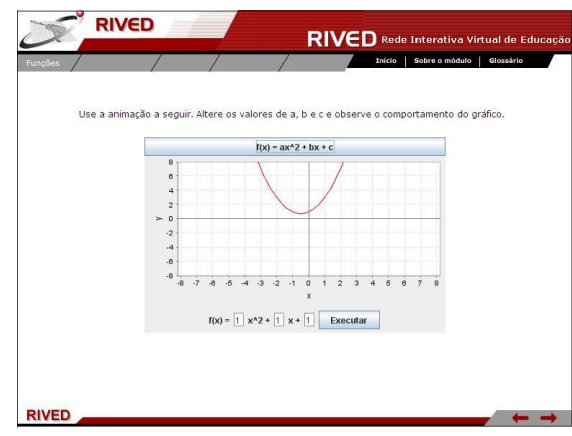

Figura 4 - Applet Java para interatividade do aluno com o objeto

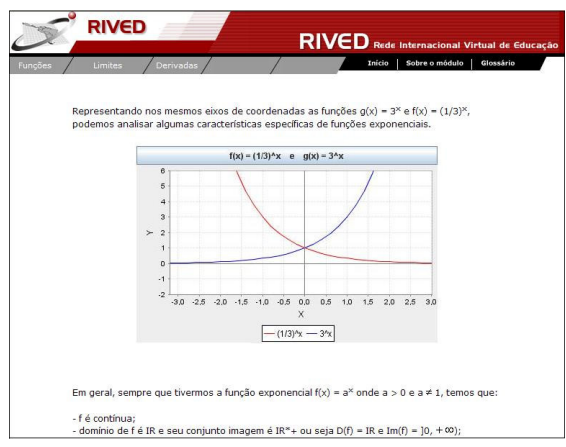

Figura 5 - Gráfico com 2 funções simultaneamente.

A navegação dentro do objeto de aprendizagem foi montada sequencialmente, de modo que o aluno pode evoluir no conteúdo de uma forma logicamente estruturada. No entanto, há menus de navegação que podem levar o aluno diretamente a um ponto desejado. Não foram aplicadas restrições na navegação. Com isso, o aluno pode seguir no conteúdo sem a necessidade da realização de testes ao final de cada tema.

Por fim, sugere-se que um objeto de aprendizagem seja acompanhado de um Guia do Professor, que é uma documentação que orienta o professor sobre o conteúdo do objeto e presta orientações quanto à ação docente neste contexto. $\mathrm{O}$ guia do professor criado para o projeto em questão está divido em duas partes: uma inicial, apresentando o objeto em si, seu objetivo, o assunto abordado, e questões técnicas e; a última, apresentando as telas componentes do objeto com instruções sobre a operação dos recursos multimídia e sugestões de abordagem em sala de aula. O guia do professor pode ser acessado por meio do link "sobre o módulo" em cada página Web do conteúdo.

\section{Resultados}

Por encaminhamentos da equipe de desenvolvimento, o objeto abordou de forma mais aprofundada o tema funções, provendo suporte a disciplina de Cálculo Diferencial e Integral I. Foi estabelecido que os objetos devam ser curtos e autocontidos, o que fez 
com que o objeto fosse segmentado em 4 módulos. São eles: Módulo 1 - funções; Módulo 2 - limites; Módulo 3 - derivadas e; Módulo4 - integrais. Restam, em aberto, os assuntos limites, derivadas e integrais para implementação futura. O referido OA ainda não foi validado cientificamente, visto que está em fase de finalização.

\section{Conclusão}

No contexto atual de globalização, as instituições de ensino devem prover aos seus acadêmicos condições de aprender por meio da utilização dos recursos disponíveis, sob pena de contribuir para uma exclusão digital e tecnológica. A utilização das TICs na educação, pela comunidade acadêmica, já é realidade. Contudo, os enfoques de Educação a Distância e de Objetos de Aprendizagem são ainda pouco utilizados, pois são recursos que devem ser constantemante pesquisados, utilizados, avaliados e aperfeiçoados. Cabe aos pesquisadores, educadores e acadêmicos, a iniciativa e a ousadia de por em prática estas ferramentas de auxílio à aprendizagem. Uma atuação no encorajamento, por organizações governamentais ou mesmo por instituições acadêmicas, pelo desenvolvimento e utilização das TIC's será decisivo para o desenvolvimento destas novas formas de aprendizagem.

O projeto RIVED atualmente provê uma forma de gerenciamento, criação e distribuição de grande parte dos objetos de aprendizagem desenvolvidos no Brasil. Há uma tendência de que com a evolução e a crescente popularização das TICs, cada vez mais instituições disponibilizem seus conteúdos educacionais pela internet, e consequentemente aumente a demanda por desenvolvimento destes recursos.

Neste cenário, o padrão SCORM se torna componente fundamental, de maneira que possibilita a criação de objetos de aprendizagem capazes de serem reaproveitados em diversos contextos. É importante destacar que não somente os objetos devem seguir uma padronização, mas também os AVAs de aprendizagem.

Da mesma forma, os recursos computacionais de desenvolvimento para ambientes Web, como o Java e o Flash, possibilitam a criação de conteúdos dinâmicos e interativos, um elo de ligação entre o aprendiz e a construção de conhecimento desejada.

Contudo, o conteúdo pedagógico deve ser cuidadosamente desenvolvido de modo que o tema abordado seja equilibrado, respeitando o intuito de auxiliar o aluno a construir os conceitos. Este aspecto é decisivo para o desenvolvimento destes recursos, pois agrupa profissionais com características distintas - educadores, pedagogos e programadores. É importante, neste caso, que a comunicação seja realizada de maneira franca e cordial, cooperativa e colaborativa. O programador deve, dentre as suas atribuições no desenvolvimento do projeto, apresentar limitações e potencialidades tecnológicas. Aos educadores e pedagogos cabem as tarefas de construir metodologias e estratégias de uso pedagógicos. A todos, segue a premissa de que deve haver o comprometimento em desenvolver, testar, antecipar, simular para que o aluno obtenha êxito na sua utilização. Afinal, um objeto de aprendizagem deve ser desenvolvido para o aluno.

Um outro aspecto que pode favorecer a popularização dos objetos de aprendizagem é a sua utilização em corporações. Este tipo de recurso para preparação de seus colaboradores, ou mesmo para exposição de seus produtos, já é realidade há algum tempo. Apesar de muitas empresas já utilizarem recursos multimídia (como apresentação de slides, animações, vídeos, etc.), elas ainda estão distantes de um desenvolvimento padronizado deste tipo de recurso e dos benefícios que isto pode gerar.

Para o futuro dos objetos de aprendizagem, entidades de pesquisa vêm trabalhando sobre uma padronização de desenvolvimento voltada para computadores de mão e 
aparelhos celulares, ou seja, dispositivos móveis em geral. A crescente demanda de conteúdo multimídia nestas modalidades da tecnologia da informação pode facilitar o acesso aos objetos de aprendizagem no futuro, sem as limitações impostas pela necessidade de um computador com conexão na Internet.

Pode se considerar que os objetos de aprendizagem, e em geral, as tecnologias envolvidas na sua produção, são um campo de estudos abrangente e que podem gerar boas oportunidades de desenvolvimento acadêmico e profissional, e ainda contribuem intrinsecamente para a inclusão digital e social. Desta forma, espera-se que iniciativas tenham cada vez mais espaço na academia e na sociedade.

\section{Referências}

ZANETTE, Elisa Netto; NICOLEIT, Evânio Ramos; GIACOMAZZO Graziela Fátima. A produção do material didático no contexto cooperativo e colaborativo da disciplina de Cálculo Diferencial e Entegral I, na modalidade de educação a distância, na graduação. In: VII CICLO DE PALESTRAS SOBRE NOVAS TECNOLOGIAS NA EDUCAÇÃ̃, 9, 2006, Porto Alegre.

TAROUCO, Liane Margarida Rockenbach; FABRE, Marie-Christine Julie Mascarenhas; TAMUSIUNAS, Fabrício Raupp. Reusabilidade de objetos educacionais. 2003. 11f. Artigo. Centro Interdisciplinar de Novas Tecnologias na Educação, Universidade Federal do Rio Grande do Sul, Porto Alegre, 2003.

WILEY, David A.. Connecting learning objects to instructional design theory: a definition, a metaphor, and a taxonomy. Utah. 2001. Disponível em: < http://www.elearning-reviews.org/topics/technology/learning-objects/2001-wileylearning-objects-instructional-design-theory/ > . Acessado em: 12 de outubro de 2006.

MACHADO, Lisandro Lemos; SILVA, Juliano Tonezer da. Objeto de aprendizagem digital para auxiliar o processo de ensino-aprendizagem no ensino técnico em informática. 2005. 16f. Artigo. Centro Interdisciplinar de Novas Tecnologias na Educação, Universidade Federal do Rio Grande do Sul, Porto Alegre, 2005.

HODGINS, Wayne; CONNER, Márcia. Everything you ever wanted to know about learning standards but were afraid to ask. 2001. Disponível em: < http://linezine.com /2.1/features/wheyewtkls.htm >. Acessado em: 14 de outubro de 2006.

GOMES, Eduardo Rodrigues. Objetos inteligentes de aprendizagem: uma abordagem baseada em agentes para objetos de aprendizagem. 2005. 99 f. Dissertação (Mestrado em Ciência da Computação) - Instituto de Informática, Universidade Federal do Rio Grande do Sul, Porto Alegre, 2005.

PASSARINI, Rosane Fátima. Objetos de aprendizagem: protótipo para módulo de ambiente de treinamento online. 2003. 105f. Dissertação (Mestrado em Ciência da Computação) - Curso de Ciência da Computação, Universidade Federal de Santa Catarina, Florianópolis, 2003.

BAILEY, Warwick. What is ADL SCORM?. 2005. 4f. Artigo. Centro de Tecnologia Educacional e Padrões de Interoperabilidade, Universidade de Bolton, Bolton, Reino Unido, 2005.

DUTRA, Renato Luís de Souza; TAROUCO, Liane Margarida Rockenbach. Objetos de aprendizagem: uma comparação entre SCORM e IMS learning design. 2006. 8f. Artigo. Centro Interdisciplinar de Novas Tecnologias na Educação, Universidade Federal do Rio Grande do Sul, Porto Alegre, 2006. 
RIVED - Rede Interativa Virtual de Educação. 2006. Disponível em: < http://rived.proinfo. mec.gov.br/ >. Acessado em: 31 de outubro de 2006.

FERREIRA, Luis de França et al. Integrando objetos de aprendizagem e realidade virtual para uso em ambientes de apoio à construção e aquisição de conhecimento e habilidade espacial. In: VII CONGRESSO IBEROAMERICANO DE INFORMÁTICA EDUCATIVA, 10, 2004, Porto Alegre.

BORGES, Francisco; NAVARRO, Mairlos. Aplicação colaborativa de objetos de aprendizagem, a partir de uma proposta de planejamento pedagógico integrado. Belo Horizonte. 2005. Disponível em: < http://portal.ibta.com.br/cursos/ibtanews/news-0106/downloads/objetos_pgl.doc >. Acessado em: 31 de outubro de 2006.

MUTINI, Samanta Patricio. Uso do padrão Instructional Management System (IMS) em objetos de aprendizagem. 2006. 125f. TCC (Bacharelado em Ciência da Computação) - Curso de Ciência da Computação, Universidade do Extremo Sul Catarinense, Criciúma, 2006.

ISOTANI, Seiji; SAHARA, Ricardo H.; BRANDÃO, Leônidas de Oliveira. iMática: ambiente interativo de apoio ao ensino de matemática via Internet. 2000. 17f. Artigo. Instituto de Matemática e Estatística, Universidade de São Paulo, São Paulo, 2000.

TAROUCO, Liane Margarida Rockenbach et al. Jogos Educacionais. 2004. 7fl. Artigo. Centro Interdisciplinar de Novas Tecnologias na Educação, Universidade Federal do Rio Grande do Sul, Porto Alegre, 2004.

ADL - Advanced Distributed Learning. 2006. Disponível em: < http://www.adl net.gov/index.cfm $>$. 